\title{
On Series of Fuzzy Numbers
}

\author{
Pishtiwan O. Sabir \\ Department of Mathematics, Faculty of Science and Science Education, University of Sulaimani, Iraq
}

\begin{abstract}
In this paper, the concepts of sequences and series of complement normalized fuzzy numbers are introduced in terms of $\gamma$-level, so that some properties and characterizations are presented, and some convergence theorems are proved.
\end{abstract}

Keywords: Fuzzy Numbers, Fuzzy Convergences, Fuzzy Series.

\section{Introduction}

Fuzzy number is one of the most important and basic conceptions in fuzzy analytics. About the conception of fuzzy number, the earliest research is Chang S.S.L's and Zadeh L.A's work [4] in 1972. After that Mizumoto and Tanaka [12], Nahmias [13], Dubois and Prade [7], Kaufmann and Gupta [9], Wang and Liu [21] successively researched the conception and properties of fuzzy number. Fuzzy numbers are of great importance in fuzzy systems. They serve as means of representing and manipulating data that are not precise but rather fuzzy, such as "about fifty years old", "real numbers close to ten", etc. On the other hand, there are some linguistic terms which cannot be represented by fuzzy numbers. Some examples [18] are "away from fifty years old", "real numbers larger than ten". Several researchers, mainly $[5,6,19,20]$, focused on computing the distance between fuzzy numbers. In [19] Tran and Duckstein introduced a distance concept based on the interval numbers where the fuzzy numbers has transformed into an interval number. The class of convergent sequences of fuzzy numbers with respect to the Hausdorff metric was first introduced by Matloka [11] in 1986 where it was established that every convergent sequence of fuzzy numbers is bounded. For sequences of fuzzy numbers, Nanda [14] in 1989, studied sequences of fuzzy numbers and showed that the set of all convergent sequences of fuzzy numbers forms a complete metric space. Later on sequences of fuzzy numbers have been discussed by Nuray et al. [15] in 1995, Savas [17] in 2000, Basarir and Mursaleen [3] in 2004, Aytar et al. [2] in 2004 and many others $[1,8,10,22]$. In section two, we first review the definitions and basic properties related to fuzzy sets and fuzzy numbers. We will also present the notations needed in the rest of the paper. In section three, the function $\tilde{\xi}$ between two complement normalized fuzzy numbers (CNFNs) is presented which plays a key role in the study of sequences and series of CNFNs. Next, some convergence theorems for sequences and series on CNFNs with respect to introduced function $\tilde{\xi}$ are proved.

\section{Preliminaries}

A fuzzy set $\tilde{A}$ defined on the universal set $X$ is a function $\mu(\tilde{A}, x): X \rightarrow[0,1]$. Frequently, we will write $\mu_{\tilde{A}}(x)$ instead of $\mu(\tilde{A}, x)$. The family of all fuzzy sets in $\mathrm{X}$ is denoted by $\mathcal{F}(X)$. The $\alpha$-level of a fuzzy set $\tilde{A}$, denoted by ${ }^{\alpha+} \tilde{A}$, is the non-fuzzy set of all elements of the universal set that belongs to the fuzzy set $\widetilde{\mathrm{A}}$ at least to the degree $\alpha \in[0,1]$. The weak $\alpha$-level ${ }^{\alpha-} \tilde{A}$ of a fuzzy set $\tilde{A} \in \mathcal{F}(X)$ is the crisp set that contains all elements of the universal set whose membership grades in the given set are greater than but do not include the specified value of $\alpha$. The largest value of $\alpha$ for which the $\alpha$-level is not empty is called the height of a fuzzy set $\tilde{A}$ denoted $\alpha_{\tilde{A}}^{\max }$. The core of a fuzzy set $\tilde{A}$ is the non-fuzzy set of all points in the universal set $\mathrm{X}$ at which $\sup _{x} \mu_{\tilde{A}}(x)$ is essentially attained. Any property of fuzzy sets that is derived from classical set theory is called a cutworthy property.

Let $\tilde{A}_{i} \in \mathcal{F}(X)$. Then the union of fuzzy sets $\tilde{A}_{i}$, denoted $\bigcup_{i} \tilde{A}_{i}$, is defined by $\mu_{\cup_{i} \tilde{A}_{i}}(x)=\sup _{x} \mu_{\tilde{A}_{i}}(x)=$ $\vee_{x} \mu_{\tilde{A}_{i}}(x)$, the intersection of fuzzy sets $\tilde{A}_{i}$, denoted $\bigcap_{i} \tilde{A}_{i}$, is defined by $\mu_{\cap_{i} \tilde{A}_{i}}(x)=\inf _{x} \mu_{\tilde{A}_{i}}(x)=\Lambda_{x} \mu_{\tilde{A}_{i}}(x)$, and the complement of $\tilde{A}_{i}$, denoted $\neg \tilde{A}_{i}$, is defined by $\mu_{\tilde{A}_{i}}(x)+\mu_{\neg \tilde{A}_{i}}(x)=1$, for all $x$ in the universal set $X$.

A fuzzy number $\tilde{a}$ is a fuzzy set defined on the set of real numbers $R^{1}$ characterized by means of a membership function $\mu_{\tilde{a}}(x): R^{1} \rightarrow[0,1]$, which satisfies: (1) $\tilde{a}$ is upper semicontinuous, (2) $\mu_{\tilde{a}}(x)=0$ outside some interval $[c, d]$, (3) There are real numbers $a, b$ such that $c \leq a \leq b \leq d$ and $\mu_{\tilde{a}}(x)$ is increasing on [c, a], $\mu_{\tilde{a}}(x)$ is decreasing on $[b, d], \mu_{\tilde{a}}(x)=1, a \leq x \leq b$. We denote the set of all fuzzy numbers by $\mathcal{F}^{\star}$.

Let $X=X_{1} \times X_{2} \times \ldots \times X_{n}$ and $f: X \rightarrow Y$ given by $y=f\left(x_{1}, x_{2}, \ldots, x_{n}\right)$. In addition, let $\tilde{A}_{1}, \tilde{A}_{2}, \ldots, \tilde{A}_{n}$ be $n$ fuzzy sets in $X_{1} \times X_{2} \times \ldots \times X_{n}$ respectively. The extension principle allows to extend the crisp function $y=f\left(x_{1}, x_{2}, \ldots, x_{n}\right)$ to act on $n$ fuzzy subsets of $X$, namely $\tilde{A}_{1}, \tilde{A}_{2}, \ldots, \tilde{A}_{n}$ such that $\tilde{B}=f\left(\tilde{A}_{1}, \tilde{A}_{2}, \ldots, \tilde{A}_{n}\right)$. Here the fuzzy set $\tilde{B}$ is defined by 


$$
\tilde{B}=\left\{\left(y, \sup _{x \in X, y=f(x)} \min \left(\mu_{\tilde{A}_{1}}\left(x_{1}\right), \mu_{\tilde{A}_{2}}\left(x_{2}\right), \ldots, \mu_{\tilde{A}_{n}}\left(x_{n}\right)\right) .\right): y=f\left(x_{1}, x_{2}, \ldots, x_{n}\right),\right.
$$

\section{Basic Definitions and Characterizations}

Throughout this section, a new fuzzy quantity, named, CNFNs and other related notions are defined and studied. Furthermore, the function $\tilde{\xi}$ between two CNFNs which plays a key role in the study of sequences and series of CNFNs has been presented. A preliminary version of this section was given in [16].

Definition 3.1. CNFN $\tilde{\mu}$ is a fuzzy set $\tilde{\mu}$ of the real line, such that core of $\neg \tilde{\mu}$ is empty and $\gamma+\neg \tilde{\mu}=\left\{\begin{array}{ll}\left\{\lambda: \mu_{\neg \tilde{\mu}}(\lambda) \geq \gamma\right\} & \text { if } \quad \gamma \in\left(0, \alpha_{\neg \widetilde{\mu}}^{\max }\right] \\ \bigcup_{0 \leq \gamma \leq \alpha_{\neg \tilde{\mu}} \max } \gamma-\neg \tilde{\mu} & \text { if } \quad \gamma=0\end{array} \quad\right.$ is compact. We use $\tilde{\mathcal{F}}_{\neg N}^{*}$ for the fuzzy power set of CNFNs.

Definition 3.2. Let $*$ denote any of the four basic arithmetic operations and let $\tilde{\mu}, \tilde{\lambda}$ be CNFNs. Then based on the extension principle we define $\tilde{\mu} \approx \tilde{\lambda}$ by

$$
\tilde{\mu} * \tilde{\lambda}=\neg\left(\neg \tilde{\mu} \uplus_{N} \neg \tilde{\lambda}\right)=\neg\left((N \neg \tilde{\mu} \circledast N \neg \tilde{\lambda})_{N}\right)
$$

Theorem 3.3. A partial ordering $=,<$, and $\preccurlyeq$ in $\tilde{\mathcal{F}}_{\neg N}^{*}$ are cutworthy.

Proof: $\neg \tilde{\lambda} \leqslant \neg \tilde{\mu}$ if and only if $N \neg \tilde{\lambda} \leqslant N \neg \tilde{\mu}$ if and only if ${ }^{\gamma+}(N \neg \tilde{\lambda}) \leq{ }^{\gamma+}(N \neg \tilde{\mu})$ if and only if $N \neg^{\gamma+} \tilde{\lambda} \leq$ $N \neg^{\gamma+} \tilde{\mu}$ if and only if $\neg^{\gamma+} \tilde{\lambda} \leq \neg^{\gamma+} \tilde{\mu}$ if and only if ${ }^{\gamma+} \tilde{\mu}^{-} \leq{ }^{\gamma+} \tilde{\lambda}^{-}$and ${ }^{\gamma+} \tilde{\mu}^{+} \leq{ }^{\gamma+} \tilde{\lambda}^{+}$. Similarly it can be shown that ${ }^{\gamma+} \tilde{\mu}^{+}<{ }^{\gamma+} \tilde{\lambda}^{-}$and ${ }^{\gamma+} \tilde{\lambda}={ }^{\gamma+} \tilde{\mu}$.

Definition 3.4. We define a function $\tilde{\xi}$ on two CNFNs $\tilde{\mu}$ and $\tilde{\lambda}$ as follows:

$$
\tilde{\xi}(\tilde{\mu}, \tilde{\lambda})=\int_{0}^{\alpha_{\tilde{n}, \tilde{\mu}}^{\max }} \gamma\left[||^{\alpha_{\tilde{\lambda}, \tilde{\mu}}^{\max }+} \tilde{\mu}^{-}-{ }^{\alpha_{\tilde{\lambda}, \tilde{\mu}}^{\max }+} \tilde{\lambda}-\left.\left|, \vee_{\gamma \leq \psi \leq \alpha_{\tilde{\lambda}, \tilde{\mu}}^{\max }}{ }^{\psi+} \tilde{\mu}^{-}-{ }^{\psi+} \tilde{\lambda}-\right| \vee\right|^{\psi+} \tilde{\mu}^{+}-{ }^{\psi+} \tilde{\lambda}^{+} \mid\right] .
$$

Theorem 3.5. Let $\tilde{\mu}, \tilde{\lambda}, \tilde{\delta}, \tilde{\psi}, \tilde{\gamma} \in \tilde{\mathcal{F}}_{\neg N}^{*}$. Then $\tilde{\xi}(\tilde{\psi}, \tilde{\delta}) \preccurlyeq \tilde{\xi}(\tilde{\mu}, \tilde{\delta}), \tilde{\xi}(\tilde{\lambda}, \tilde{\psi}) \preccurlyeq \tilde{\xi}(\tilde{\mu}, \tilde{\delta})$ and $\tilde{\xi}(\tilde{\mu}, \tilde{\psi}) \preccurlyeq \tilde{\xi}(\tilde{\mu}, \tilde{\delta})$ whenever $\tilde{\mu} \preccurlyeq \tilde{\lambda} \preccurlyeq \tilde{\delta}$, and $\tilde{\mu} \preccurlyeq \tilde{\psi} \preccurlyeq \tilde{\delta}$.

Proof: By hypothesis we have, $\left|{ }^{\psi+} \tilde{\lambda}^{-}-{ }^{\psi+} \tilde{\psi}^{-}\right| \vee\left|{ }^{\psi+} \tilde{\lambda}^{+}-{ }^{\psi+} \tilde{\psi}^{+}\right| \leq\left.\left|{ }^{\psi+} \tilde{\mu}^{-}-{ }^{\psi+} \tilde{\delta}^{-}\right| \vee\right|^{\psi+} \tilde{\mu}^{+}-{ }^{\psi+} \tilde{\delta}^{+} \mid$. So that, $\vee_{\gamma \leq \psi \leq \alpha} \alpha_{\bar{\lambda}, \tilde{\psi}}\left|{ }^{\psi+} \tilde{\lambda}^{-}-{ }^{\psi+} \tilde{\psi}^{-}\right| \vee\left|{ }^{\psi+} \tilde{\lambda}^{+}-{ }^{\psi+} \tilde{\psi}^{+}\right| \leq \mathrm{V}_{\gamma \leq \psi \leq \alpha} \max \tilde{\bar{\lambda}, \tilde{\mu}}\left|{ }^{\psi+} \tilde{\mu}^{-}-{ }^{\psi+} \tilde{\delta}^{-}\right| \vee\left|{ }^{\psi+} \tilde{\mu}^{+}-{ }^{\psi+} \tilde{\delta}^{+}\right|$. Also,

$\left|{ }^{\psi+} \tilde{\mu}^{-}-{ }^{\psi+} \tilde{\psi}^{-}\right| \leq\left.\right|^{\psi+} \tilde{\mu}^{-}-{ }^{\psi+} \tilde{\delta}^{-} \mid$and $\left|{ }^{\psi+} \tilde{\mu}^{+}-{ }^{\psi+} \tilde{\psi}^{+}\right| \leq\left|{ }^{\psi+} \tilde{\mu}^{+}-{ }^{\psi+} \tilde{\delta}^{+}\right|$, which implies that

$\left.\left|{ }^{\psi+} \tilde{\mu}^{-}-{ }^{\psi+} \tilde{\psi}^{-}\right| \vee\right|^{\psi+} \tilde{\mu}^{+}-{ }^{\psi+} \tilde{\psi}^{+}|\leq|{ }^{\psi+} \tilde{\mu}^{-}-\left.{ }^{\psi+} \tilde{\delta}^{-}|\vee|\right|^{\psi+} \tilde{\mu}^{+}-{ }^{\psi+} \tilde{\delta}^{+} \mid$, and

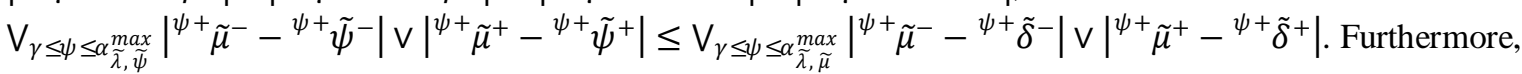

$\left|{ }^{\psi+} \tilde{\delta}^{-}-{ }^{\psi+} \tilde{\psi}^{-}\right| \leq\left|{ }^{\psi+} \tilde{\mu}^{-}-{ }^{\psi+} \tilde{\delta}^{-}\right|$and $\left|{ }^{\psi+} \tilde{\delta}^{+}-{ }^{\psi+} \tilde{\psi}^{+}\right| \leq\left|{ }^{\psi+} \tilde{\mu}^{+}-{ }^{\psi+} \tilde{\delta}^{+}\right|$, implies $\left.\quad\right|^{\psi+} \tilde{\delta}^{-}-{ }^{\psi+} \tilde{\psi}^{-} \mid \vee$ $\left|{ }^{\psi+} \tilde{\delta}^{+}-{ }^{\psi+} \tilde{\psi}^{+}\right| \leq\left|{ }^{\psi+} \tilde{\mu}^{-}-{ }^{\psi+} \tilde{\delta}^{-}\right| \vee\left|{ }^{\psi+} \tilde{\mu}^{+}-{ }^{\psi+} \tilde{\delta}^{+}\right|$. Hence,

$\left.\vee_{\gamma \leq \psi \leq \alpha_{\bar{\lambda}, \tilde{\psi}}}\right|^{\psi+} \tilde{\delta}^{-}-{ }^{\psi+} \tilde{\psi}^{-}|\vee|{ }^{\psi+} \tilde{\delta}^{+}-{ }^{\psi+} \tilde{\psi}^{+}\left|\leq \mathrm{V}_{\gamma \leq \psi \leq \alpha_{\bar{\lambda}, \tilde{\mu}}^{\max }}\right|^{\psi+} \tilde{\mu}^{-}-{ }^{\psi+} \tilde{\delta}^{-}|\vee|{ }^{\psi+} \tilde{\mu}^{+}-{ }^{\psi+} \tilde{\delta}^{+} \mid$.

Definition 3.6. Let $\left(\tilde{\mu}_{\eta}\right)$ be fuzzy sequence in $\tilde{\mathcal{F}}_{\neg N}^{*}$ and $\tilde{\mu} \in \tilde{\mathcal{F}}_{\neg N}^{*}$. We say that $\left(\tilde{\mu}_{\eta}\right)$ is $\tilde{\xi}$-converges to $\tilde{\mu}$ written as $\left(\tilde{\mu}_{\eta}\right) \stackrel{\tilde{\xi}}{\longrightarrow} \tilde{\mu}$, if for any $\varepsilon>0$, there exists a positive integer $N$ such that $\tilde{\xi}\left(\tilde{\mu}_{\eta}, \tilde{\mu}\right) \prec \varepsilon$ as $\eta \geq N$.

Definition 3.7. Let $\left(\tilde{\mu}_{\eta}\right)$ be fuzzy sequence in $\tilde{\mathcal{F}}_{\neg N}^{*}$ and $\tilde{\mu} \in \tilde{\mathcal{F}}_{\neg N}^{*}$. We say that $\left(\tilde{\mu}_{\eta}\right)$ is $\gamma$-level converges to $\tilde{\mu}$ written as $\left(\tilde{\mu}_{\eta}\right) \stackrel{\gamma-\text { level }}{\longrightarrow} \tilde{\mu}$, if ${ }^{\gamma+} \tilde{\mu}_{\eta}{ }^{-} \longrightarrow{ }^{\gamma+} \tilde{\mu}^{-}$and $^{\gamma+} \tilde{\mu}_{\eta}{ }^{+} \longrightarrow{ }^{\gamma+} \tilde{\mu}^{+}$for all $\gamma \in\left[0, \alpha_{\tilde{\mu}, \tilde{\mu}_{\eta}}^{\max }\right]$.

Theorem 3.8. $\left(\tilde{\mu}_{\eta}\right)$ be fuzzy sequence in $\tilde{\mathcal{F}}_{\neg N}^{*}$ and $\tilde{\mu} \in \tilde{\mathcal{F}}_{\neg N}^{*}$. Then $\left(\tilde{\mu}_{\eta}\right) \stackrel{\tilde{\xi}}{\longrightarrow} \tilde{\mu}$ if and only if $\left(\tilde{\mu}_{\eta}\right)$ is $\gamma$-level converges to $\tilde{\mu}$.

Proof: Obvious.

Theorem 3.9. Let $\left(\tilde{\mu}_{\eta}\right),\left(\tilde{\lambda}_{\eta}\right),\left(\tilde{\psi}_{\eta}\right) \subset \tilde{\mathcal{F}}_{\neg N}^{*}$ and $\tilde{\mu}, \tilde{\lambda}, \tilde{\psi} \in \tilde{\mathcal{F}}_{\neg N}^{*}, \quad * \in\{+,-, ; /\}$. If $\left(\tilde{\mu}_{\eta}\right) \stackrel{\gamma-\text { level }}{\longrightarrow} \tilde{\mu}$ and $\left(\tilde{\lambda}_{\eta}\right) \stackrel{\gamma \text {-level }}{\longrightarrow} \tilde{\lambda}$, then

1. $\left(\tilde{\mu}_{\eta}\right) \uplus\left(\tilde{\lambda}_{\eta}\right) \stackrel{\gamma \text {-level }}{\longrightarrow} \tilde{\mu}$ 团 $\tilde{\lambda}$ such that $0 \notin^{0-} \tilde{\lambda}_{\eta}\left(\right.$ resp. $\left.^{0-} \tilde{\lambda}\right)$ in the case $*=/$.

2. $\tilde{\mu}=\tilde{\lambda}$ where $\tilde{\mu}_{\eta}=\tilde{\lambda}_{\eta}$. Also $\tilde{\mu} \leqslant \tilde{\psi}$ where $\tilde{\mu}_{\eta} \leqslant \tilde{\psi}$.

3. $\left(\tilde{\psi}_{\eta}\right) \stackrel{\gamma \text {-level }}{\longrightarrow} \tilde{\mu}$ where $\tilde{\mu}=\tilde{\lambda}$ and $\tilde{\mu}_{\eta} \preccurlyeq \tilde{\psi}_{\eta} \preccurlyeq \tilde{\lambda}_{\eta}$.

Proof: We only prove (1) for the case $*=+$, the proof of the other part are similar. By hypothesis, ${ }^{\gamma+} \tilde{\mu}_{\eta}{ }^{-} \longrightarrow{ }^{\gamma+} \tilde{\mu}^{-}, \quad{ }^{\gamma+} \tilde{\mu}_{\eta}{ }^{+} \longrightarrow{ }^{\gamma+} \tilde{\mu}^{+},{ }^{\gamma+} \tilde{\lambda}_{\eta}{ }^{-} \longrightarrow{ }^{\gamma+} \tilde{\lambda}^{-},{ }^{\gamma+} \tilde{\lambda}_{\eta}{ }^{+} \longrightarrow{ }^{\gamma+} \tilde{\lambda}^{+}$. Therefore, ${ }^{\gamma+} \tilde{\mu}_{\eta}{ }^{-}+$ 


${ }^{\gamma+} \tilde{\lambda}_{\eta}{ }^{-} \longrightarrow{ }^{\gamma+} \tilde{\mu}^{-}+{ }^{\gamma+} \tilde{\lambda}^{-} \quad$ and $\quad{ }^{\gamma+} \tilde{\mu}_{\eta}{ }^{+}+{ }^{\gamma+} \tilde{\lambda}_{\eta}{ }^{+} \longrightarrow^{\gamma+}{ }^{\gamma+} \tilde{\mu}^{+}+{ }^{\gamma+} \tilde{\lambda}^{+}$.
${ }^{\gamma+}\left(\tilde{\mu}_{\eta}+\tilde{\lambda}_{\eta}\right)^{-} \longrightarrow{ }^{\gamma+}(\tilde{\mu}+\tilde{\lambda})^{-}$and ${ }^{\gamma+}\left(\tilde{\mu}_{\eta}+\tilde{\lambda}_{\eta}\right)^{+} \longrightarrow{ }^{+}(\tilde{\mu}+\tilde{\lambda})^{+}$.

\section{Fuzzy Series On CNFNs}

In this section, many results of fuzzy series of CNFNs based on $\gamma$-level converges are examined.

Definition 4.1. Let $\left(\tilde{\mu}_{n}\right)$ be a sequence of CNFNs, and $\tilde{\lambda}_{n}=\tilde{\mu}_{1} \boxplus \tilde{\mu}_{2} \boxplus \tilde{\mu}_{3} \boxplus \ldots \boxplus \tilde{\mu}_{n}$. If the sequence $\left(\tilde{\lambda}_{n}\right) \gamma$ level converges to $\tilde{\mu}$, then we say that the fuzzy series $\widetilde{\sum_{k=1}^{\infty}} \tilde{\mu}_{k}=\tilde{\mu}_{1} \boxplus \tilde{\mu}_{2} \boxplus \tilde{\mu}_{3} \boxplus \ldots \boxplus \tilde{\mu}_{n} \boxplus \ldots$ of CNFNs $\tilde{\xi}$ converge to $\tilde{\mu}$.

Theorem 4.2. Let $\tilde{\mu} \in \tilde{\mathcal{F}}_{\neg N}^{*},\left(\tilde{\mu}_{n}\right) \subset \tilde{\mathcal{F}}_{\neg N}^{*}$ then $\widetilde{\sum_{k=1}^{\infty} \tilde{\mu}_{k}}=\tilde{\mu}$ if and only if $\sum_{k=1}^{\infty}{ }^{\gamma+} \tilde{\mu}_{k}={ }^{\gamma+} \tilde{\mu}$ for all $\gamma \in$ $\left[0, \alpha_{\tilde{\mu}}^{\max }\right]$.

Proof: Consider $\tilde{\lambda}_{n}=\tilde{\mu}_{1} \boxplus \tilde{\mu}_{2} \boxplus \tilde{\mu}_{3} \boxplus \ldots \boxplus \tilde{\mu}_{n}$. Then $\tilde{\lambda}_{n} \stackrel{\gamma \text {-level }}{\longrightarrow} \tilde{\mu}$ if and only if ${ }^{\gamma+} \tilde{\lambda}_{n}{ }^{-} \longrightarrow{ }^{\gamma+} \tilde{\mu}^{-}$and ${ }^{\gamma+} \tilde{\lambda}_{n}{ }^{+} \longrightarrow{ }^{\gamma+} \tilde{\mu}^{+}$if and only if ${ }^{\gamma+} \tilde{\lambda}_{n}{ }^{-}=\sum_{k=1}^{n}{ }^{\gamma+} \tilde{\mu}_{k}{ }^{-}$and ${ }^{\gamma+} \tilde{\lambda}_{n}{ }^{+}=\sum_{k=1}^{n}{ }^{\gamma+} \tilde{\mu}_{k}{ }^{+}$if and only if $\sum_{k=1}^{\infty}{ }^{\gamma+} \tilde{\mu}_{k}=$ $\gamma+\mu$.

Corollary 4.3. Let $\tilde{\mu} \in \tilde{\mathcal{F}}_{\neg N}^{*}, \tilde{\mu}_{n} \subset \tilde{\mathcal{F}}_{\neg N}^{*}$ then $\widetilde{\sum_{k=1}^{\infty}} \tilde{\mu}_{k}=\tilde{\mu}$ if and only if $\sum_{k=1}^{\infty}{ }^{\gamma+} \tilde{\mu}_{k}{ }^{+}={ }^{\gamma+} \tilde{\mu}^{+}$and $\sum_{k=1}^{\infty}{ }^{\gamma+} \tilde{\mu}_{k}{ }^{-}=$ $\gamma+\mu^{-}$.

Proof: Obvious.

Theorem 4.4. Let $\tilde{\mu}, \tilde{\lambda} \in \tilde{\mathcal{F}}_{\neg N}^{*}, \tilde{\mu}_{n}, \tilde{\lambda}_{n} \subset \tilde{\mathcal{F}}_{\neg N}^{*}, \widetilde{\sum_{k=1}^{\infty}} \tilde{\mu}_{k}=\tilde{\mu}$ and $\widetilde{\sum_{k=1}^{\infty} \tilde{\lambda}_{k}}=\tilde{\lambda}$, then $\sum_{k=1}^{\infty}{ }^{\gamma+}\left(\tilde{\mu}_{k} \text { * } \tilde{\lambda}_{k}\right)^{+}=$ ${ }^{\gamma+}(\tilde{\mu} * \tilde{\lambda})^{+}$and $\sum_{k=1}^{\infty}{ }^{\gamma+}\left(\tilde{\mu}_{k} * \tilde{\lambda}_{k}\right)^{-}={ }^{\gamma+}(\tilde{\mu} * \tilde{\lambda})^{-}$.

Proof: Consider $\tilde{\beta}_{n}=\widetilde{\sum_{k=1}^{n}} \tilde{\mu}_{k}$ and $\tilde{\psi}_{n}=\widetilde{\sum_{k=1}^{n}} \tilde{\lambda}_{k}$. Then $\tilde{\beta}_{n} \stackrel{\gamma \text {-level }}{\longrightarrow} \tilde{\mu}$ and $\tilde{\psi}_{n} \stackrel{\gamma \text {-level }}{\longrightarrow} \tilde{\lambda}$. Implies $\tilde{\beta}_{n}$ * $\tilde{\psi}_{n} \stackrel{\gamma-\text { level }}{\longrightarrow} \tilde{\mu}$ * $\tilde{\lambda}$. This implies that ${ }^{\gamma+}\left(\tilde{\beta}_{n} * \tilde{\psi}_{n}\right) \stackrel{\gamma-\text { level }}{\longrightarrow}^{\gamma+}(\tilde{\mu}$ * $\tilde{\lambda})$. So that $\sum_{k=1}^{\infty}{ }^{\gamma+}\left(\tilde{\mu}_{k} * \tilde{\lambda}_{k}\right)=$ ${ }^{\gamma+}(\tilde{\mu} \uplus \tilde{\lambda})$.

Theorem 4.5. If $\left(\tilde{\mu}_{\eta}\right),\left(\tilde{\lambda}_{\eta}\right) \subset \tilde{\mathcal{F}}_{\neg N}^{*}$ that has the properties $\chi_{\widetilde{\mu}_{\eta}}\left(\mu_{\eta}\right)=0=\chi_{\widetilde{\lambda}_{\eta}}\left(\lambda_{\eta}\right)$ for every $\lambda_{\eta}$ and $\mu_{\eta}$ less than zero and $\mathrm{V}_{0 \leq \gamma \leq \alpha_{\tilde{\mu}_{\eta}}^{\max }}{ }^{\gamma+} \tilde{\mu}_{k} \leq \mathrm{V}_{0 \leq \gamma \leq \alpha_{\tilde{\mu}_{\eta}}^{\max }}{ }^{\gamma+} \tilde{\lambda}_{k}$ for all $k$ sufficiently large. Then $\widetilde{\sum_{k=1}^{\infty}} \tilde{\mu}_{k}$ is $\tilde{\xi}$-converge whenever $\widetilde{\sum_{k=1}^{\infty}} \tilde{\lambda}_{k}$ is $\tilde{\xi}$-converges.

Proof: Obvious.

\section{References}

Altin, Y., Et, M. and Basarir, M., On some generalized difference sequences of fuzzy numbers, Kuwait J. Sci. Eng. 34 (2007) 1-14. Aytar, S., Statistical limit points of sequences of fuzzy numbers, Inf. Sciences 165 (2004) 129-138.

Basarir, M., and Mursaleen, M., Some differemce sequence spaces of fuzzy numbers, J. of Fuzzy Math., 12 (2004) 1 -6.

Chang, S. S. L., Zadeh, L. A., Fuzzy mappings and control, IEEE Trans. Syst., Man and Cyber., SMC-2, (1972) 30-34.

Cheng, C. H., A new approach for ranking fuzzy numbers by distance method, Fuzzy Sets and Systems 95 (1998) 307-317.

Diamond, P., Fuzzy least squares, Information Sciences 46 (1988) 141-157.

Dubois, D. and Prade, H., Operations on fuzzy numbers, Int. J. Systems Sci. 9 (1978) 613-626.

Fang, J. X. and Huang, H., On the level convergence of a sequence of fuzzy numbers, Fuzzy Sets and Systems 147 (2004) 417-435 Kaufmann, A., and Gupta, M. M., Introduction to Fuzzy Arithmetic: Theory and Applications, Van Nostand Reinhold, NY, 1991.

Kavikumar, J., Khamis, A. B., and Kandasamy, R., Fuzzy entire sequence spaces, Int. J. of Math. and Math. Sci. (2007), doi: $10.1155 / 2007 / 58368$.

Matloka, M., Sequences of fuzzy numbers, Bulletin for Studies and Exchanges on Fuzziness and its Applications, 28 (1986) $28-37$. Mizumoto, M., and Tanaka, K., The four operations of arithmetic on fuzzy numbers, Systems Computers Controls 5 (1976) 73-81.

Nahmias, S., Fuzzy variables, Fuzzy Sets and Systems 1 (1978) 97-110.

Nanda, S., On sequences of fuzzy numbers, FSS 33 (1989) 123-126.

Nuray, F., and Savas, E., Statistical convergence of fuzzy numbers, Mathematica Slovaca 30 (1995) 269-273.

Sabir, P. O., Jabbar, A. K., and Al-Khafagi, M. A., On Fuzzy Numbers and Fuzzy Convergence of Fuzzy Sequence, Journal of Koya University 26 (2013) 33-56.

Savas, E., A note on sequence of fuzzy numbers, Info. Sciences 124 (2000) 297-300.

Taheri, S. M., c-fuzzy numbers and a dual of extension principle, Information Sciences 178 (2008) 827-835.

Tran, L., and Duckstein, L., Comparision of fuzzy numbers using a fuzzy distance measure, FSS 130 (2002) 331-341.

Voxman, W., Some remarks on distances between fuzzy numbers, Fuzzy Sets and Systems 100 (1998) 353-365.

Wang, G. and Liu, R., The properties of fuzzy number valued power function, Proc. of the $5^{\text {th }}$ ICMLC, Dalian, (2006) 1679-1684.

Wang, G. and Xi, X., Convergence of sequences on the fuzzy real line, Fuzzy Sets and Systems 127 (2002) 323-331. 